\title{
Influência do ambiente físico de trabalho na criação do conhecimento nas organizações
}

Ana Paula Graciola

Graduação em Arquitetura e Urbanismo, Universidade de Caxias do Sul. Aluna do Mestrado Acadêmico em Administração, Universidade de Caxias do Sul

Suélen Bebber

Aluna do Mestrado Acadêmico em Administração, Universidade de Caxias do Sul

Fernanda Pauletto D'Arrigo

Graduação em Arquitetura e Urbanismo, Universidade de Caxias do Sul. Aluna do Mestrado Acadêmico em Administração, Universidade de Caxias do Sul

Ana Cristina Fachinelli

Programa de Pós-Graduação em Administração, Universidade de Caxias do Sul. Docente do Programa de Pos Graduação em Administração, Universidade de Caxias do Sul

Gabriel Sperandio Milan

Docente do Programa de Pós-Graduação em Administração, Universidade de Caxias do Sul

Deonir de Toni

Docente do Programa de Pós-Graduação em Administração, Universidade de Caxias do Sul

http://dx.doi.org/10.1590/1981-5344/2407

O ambiente de trabalho pode contribuir para o desempenho e produtividade dos trabalhadores bem como para a troca de informações entre colegas de trabalho que, ao interagirem, promovem a criação de conhecimento. O objetivo do presente estudo foi o de identificar a influência da satisfação com os aspectos tangíveis do ambiente construído sobre os aspectos intangíveis do processo de criação do conhecimento em 
empresas do Rio Grande do Sul. O método adotado foi de natureza quantitativa com abordagem descritiva. Os dados foram coletados por meio de uma survey com corte transversal. O campo de estudo foi o das áreas intensivas em conhecimento de empresas. Para a análise dos dados foram adotadas técnicas de estatística multivariada. Os resultados revelaram a relação positiva entre a criação de conhecimento tácito e a satisfação com o ambiente construído. Mais especificamente, os resultados indicam que a satisfação com o ambiente construído apresenta maior impacto sobre a criação de conhecimento, nas áreas da tecnologia e aprendizagem.

Palavras-chave: Ambiente construído; Satisfação com o ambiente construído; Criação do conhecimento.

\section{Work built environment influence on organizational knowledge creation}

The work environment can contribute for the performance and productivity of workers as well as information transfer among coworkers that, when interacting, make that the knowledge creation happens. Therefore, the objective is to identify the influence of the satisfaction with the built environment on the knowledge creation process in knowledge intense companies of Rio Grande do Sul, Brazil. A survey research was carried out with a structured questionnaire based on theoretical references related to the study context, and had as target public companies of Rio Grande do Sul that have as common characteristic, intense areas of knowledge. Such research, of exploratory and quantitative feature, had data analyses done with the application of multivariate statistics technics. The results show from the four areas that represent knowledge creation, in this study, built environment satisfaction presented better influence on knowledge creation, which is, better built environments increases information exchange through the use of technology.

Keywords: Built environment; Built environment satisfaction; Knowledge creation.

Recebido em24.04.2015 Aceito em19.02.2016 


\section{Introdução}

A relação entre o ambiente físico das organizações e as interações pessoais é percebida como uma forma de aumentar o desempenho e as inovações das organizações. Por esta razão, as empresas passam a investir na construção de ambientes de trabalho que estimulem a criatividade e a interação entre seus membros (WABER; MAGNOLFI; LINDSAY, 2014). O Facebook, por exemplo, une milhares de funcionários em uma única sala de um quilômetro e meio de extensão. Já a Yahoo abandonou a ideia de trabalho a distância, por privilegiar as conversas informais nos corredores e refeitórios. A Samsung quebra a hierarquia tradicional existente e projeta grandes áreas abertas, situadas entre os andares onde a empresa espera que os engenheiros e vendedores se misturem, e que assim haja colisão de ideias inovadoras entre as pessoas (WABER; MAGNOLFI; LINDSAY, 2014). São casos que adotam estruturas não convencionais de trabalho. Os espaços privados, os cubículos, foram transformados em espaços abertos, o que possibilita o convívio entre pessoas extrovertidas e introvertidas, no mesmo ambiente de trabalho (WABER; MAGNOLFI; LINDSAY, 2014).

Neste contexto a satisfação com o ambiente de trabalho resulta em melhor desempenho dos funcionários, fator importante para o sucesso das organizações (DOLE; SCHROEDER, 2001). Além do mais, o ambiente construído está diretamente relacionado com a satisfação dos trabalhadores, com sua produtividade e com o nível de competitividade das empresas (HESKETT et al., 1994; VAN DER VOORDT, 2004; LEE, 2006) e indiretamente relacionado ao compromisso com a organização e a troca de emprego (CARLOPIO, 1996).

Infinitos corredores nas empresas são substituídos por ambientes que promovem os encontros entre as pessoas. Projetar espaços não depende apenas da estruturação de ambientes para se viver, trabalhar e estudar. Depende também da compreensão de como se vive, trabalha e estuda nestes ambientes pois são espaços que influenciam no comportamento daqueles que circulam nestes locais. A composição física dos espaços, os projetos arquitetônicos, dependem da estrutura organizacional. Consoante isso, cada empresa necessita equilibrar e adaptar as necessidades da estrutura organizacional aos espaços, com o intuito de gerar interação e trocas de informações entre os grupos e indivíduos (ALLEN; HENN, 2007). Assim, espaços organizacionais são responsáveis pela transferência de informações e é onde a criação do conhecimento acontece (CONGDON; FLYNN; REDMAN, 2014).

Portanto, o objetivo do presente estudo foi identificar a influência da satisfação com os aspectos tangíveis do ambiente construído, sobre os aspectos intangíveis do processo de criação do conhecimento em empresas intensivas em conhecimento do Rio Grande do Sul. Nas organizações estudadas, o conhecimento desempenha um papel central 
na criação, uso, desenvolvimento, gerenciamento ou comercialização dos produtos ou serviços. De acordo com Gupta e Sharma (2004), as organizações intensivas em conhecimento, reúnem conceitos de alta qualidade técnica intimamente relacionados com a aprendizagem organizacional (LEVITT; MARCH, 1988), trabalhadores do conhecimento (DRUCKER, 1999), capital intelectual (EDVINSSON; MALONE, 1998; STEWART, 1998) e gestão do conhecimento (KOGUT; ZANDER, 1992; NONAKA; TAKEUCHI, 1995). Para cumprir com o objetivo proposto, o estudo foi construído a partir de pesquisa sobre a satisfação com o ambiente construído em relação à criação de conhecimento nas organizações.

\section{Marco teórico}

Diferentes estudos avaliaram o efeito negativo de ambientes mal projetados e a Síndrome do Edifício Doente (SED) (Sick building syndrome) (HEDGE; STERLING; STERLING, 1986; BURGE et al., 1987; MOLHAVE, 1987; VALJBORN, 1989; NORBACK; MICHEL; WIDSTROM, 1990; RYAN; MORROW, 1992; GUPTAA; KHAREB; GOYALC, 2007; KIM et al., 2013). Esta síndrome consiste na perturbação do desempenho dos trabalhadores, pacientes ou usuários dos ambientes, com o declínio da produtividade, com surgimento de doenças e a sensação de mal estar em decorrência da exaustão emocional e insatisfação com os ambientes projetados. Deste modo, foi constatada a crescente consciência da significante relação existente entre a qualidade do ambiente interno e a SED (FISK, 2000; JONES, 1999; WARGOCKI, 2000). Assim, quanto maior a satisfação com a qualidade do ambiente construído maior a produtividade e o desempenho dos usuários do edifício (LEAMAN; BORDASS, 2007; HUMPHREYS; NICOL, 2007).

Kim et al. (2013) ao investigarem a percepção dos ocupantes de edifícios acerca da qualidade do ambiente construído, realizaram uma revisão de literatura que contemplou fatores como a qualidade do ar, a síndrome do edifício doente, o conforto térmico, o conforto luminoso, bem como o conforto acústico, o layout e mobiliário dos escritórios, e a limpeza relacionado aos ambientes, sendo estes, portanto, fatores que determinam a qualidade do ambiente construído. No âmbito da satisfação com os ambientes construídos, uma edificação adequada ao uso mantem a harmonia suficiente com relação à percepção humana que engloba a visão, audição, odores e como o espaço é sentido pelo usuário. Deste modo, é preciso compreender a acessibilidade geral, a eficiência funcional, sua flexibilidade, segurança e orientação espacial (VAN DER VOORDT; VAN WEGEN, 2013).

A dimensão física dos espaços relacionais tem sido estudada no âmbito organizacional também no contexto da inovação e dos fluxos de informação e conhecimento (ALLEN 1977; ALLEN; HENN, 2007). A interface especificamente com o campo da gestão do conhecimento se dá pela questão da interação entre as pessoas no ambiente organizacional. A 
criação do conhecimento tem sido discutida nas organizações contemporâneas (CHOU; WANG, 2003) também na perspectiva da interação entre as pessoas no ambiente pois para compreender a dinâmica social da criação do conhecimento, é preciso identificar a forma como as trocas de informações acontecem, bem como as condições para criação do conhecimento (NONAKA; TAKEUCHI, 1995; NONAKA; TOYAMA; HIRATA, 2011). Adquirir, assimilar, transformar e utilizar o conhecimento são dimensões de um processo de absorção do conhecimento pelo indivíduo, que só acontece por meio do relacionamento do individuo com a sociedade (ZAHRA; GEORGE, 2002). Assim, compreende-se que a capacidade de perceber o conhecimento e transformá-lo em um novo conhecimento está diretamente relacionada à interação.

Interagir é a fonte da criação do conhecimento, este que é subjetivo, estético, adquirido com a prática e apresenta relação com os processos. No entanto, há teóricos que insistem em excluir a subjetividade da gestão para a construção teórica objetiva, através da eliminação das diferenças, com a criação de padrões. Entretanto, são estas diferentes perspectivas, capacidades e visões que propulsionam a criação de novos conhecimentos, nos ambientes corporativos (NONAKA; TOYAMA; HIRATA, 2011).

Na criação do conhecimento organizacional, os indivíduos interagem com os demais para transcender suas próprias barreiras, mudam a si mesmos, os demais, a organização e o ambiente em que vivem. Portanto, o conhecimento é criado socialmente na síntese de diferentes visões de várias pessoas (NONAKA; TOYAMA; HIRATA, 2011). Deste modo, o conhecimento nasce da subjetividade de cada indivíduo, inserido num contexto com o encontro da objetividade através de processos sociais de trocas de informações, ou seja, é a interação do conhecimento tácito de cada indivíduo (subjetivo) com o conhecimento explícito (objetivo) e, juntos, são fontes para a criação de conhecimento (ALAVI; LEIDNER, 2001; NONAKA; TOYAMA; HIRATA, 2011). É através da distribuição de informação, ou o fluxo de informação, que o conhecimento é adquirido (ALLEN, 1977; HUBER, 1991; CHOU; WANG, 2003).

Nonaka e Konno (1998) compreendem que a criação do conhecimento é gerada através do "ba" de cada organização, também traduzido como espaço. No entanto, esta tradução é melhor compreendida com as relações nos espaços físicos, virtuais ou mentais. Para Nonaka e Takeuchi (1995), existem quatro maneiras para que a criação do conhecimento aconteça, através da socialização, da internacionalização, da externacionalização e a combinação destas; em um ambiente em que os individuos possam se comunicar e trocar experiências cara a cara, que ocorra interação, para contribuir com a externacionalização da criação do conhecimento. A interação em um ambiente é necessária para promover o conhecimento tácito que é convertido em conhecimento explícito, e é assim compartilhado entre os membros da organização. O ba, desse modo, rompe a ideia de que a criação de conhecimento é individual, descontextualizada, autônoma e que desconsidera as interações humanas, 
pois, na verdade, é um processo dinâmico e comunicativo que ultrapassa os limites do individuo e da empresa, e se concretiza através de uma plataforma onde se utiliza uma mesma linguagem em busca de objetivos comunitários (FAYARD, 2010).

A criação de conhecimento desempenha papel crucial nas empresas e, portanto, emerge uma questão fundamental sobre o tema acerca de quais são os processos que facilitam a criação do conhecimento ( $\mathrm{CHOU}$; WANG, 2003). Isto posto, o estudo de Chou e Wang (2003), tendo em vista que processos intencionais de criação de conhecimento podem ser encorajados através de intervenções gerenciais, objetivou identificar tais intervenções gerenciais que podem contribuir para o processo de criação do conhecimento. Assim, os autores examinaram o impacto que 0 gerenciamento da informação e as atividades de aprendizagem exercem na criação do conhecimento. O estudo identificou a conexão entre os tipos de criação do conhecimento e os vários bas organizacionais, o que contribui de forma significativa para estudos empíricos, já que o estudo de Nonaka e Takeuchi (1995) forneceu apenas embasamento teórico sobre a aquisição do conhecimento.

De acordo com Fayard (2010) o processo de criação do conhecimento descrito por Nonaka, é relativo, visto que depende da especificidade dos contextos, das partes envolvidas e dos objetivos perseguidos. Por isso, o processo de criação do conhecimento pode ser qualificado como operacional ou mesmo estratégico. O ba é imprecisamente orientado e favorece o surgimento coletivo de novos conhecimentos a partir da participação de indivíduos confiantes e, as relações que acontecem dentro de um $b a$, evoluem de maneira flexível e aberta.

Neste cenário, a curva de Allen (1977) se mantém atualizada para a realidade que vivemos, estudo que comprova a forte correlação negativa existente entre a distância física e a potencialidade e/ou a frequência de comunicação entre as pessoas. A estimativa é que ocorra comunicação regularmente com pessoas que estejam próximas, sentadas a 1,8 metros de distância, do que com colegas que trabalham em diferentes andares ou em ambientes com grandes distâncias, como 18 metros. Deste modo, a medida que a tecnologia encurta distâncias, a proximidade é fundamental para que haja interação entre as pessoas, consequentemente a comunicação aumenta com a diminuição da distância. Portanto, o layout organizacional é um forte determinante da escolha do tipo de comunicação empresarial (ALLEN, 1977; ALLEN; HENN, 2007; WABER; MAGNOLFI; LINDSAY, 2014). Além do mais, é possível constatar que quanto maior a complexidade das informações, a comunicação cara a cara promove o feedback (ALLEN; HENN, 2007) necessário para que as trocas de informações aconteçam (WABER; MAGNOLFI; LINDSAY, 2014).

\section{Método}


O método proposto para o desenvolvimento da pesquisa é de natureza quantitativo-descritiva (HAIR Jr. et al., 2010); MALHOTRA; BIRKS; WILLS, 2012) que foi implementada por meio de um levantamento, ou seja, da aplicação de uma survey com corte transversal (FOWLER Jr., 2009; HAIR Jr. et al., 2010; REMLER; VAN RYZIN, 2011). A aplicação de uma pesquisa do tipo survey se dá por meio de um instrumento de coleta de dados (questionário), em uma amostra representativa de uma população-alvo e destinada a gerar informações específicas dos entrevistados (HAIR Jr. et al., 2010; MALHOTRA; BIRKS; WILLS, 2012; FINK, 2013).

A pesquisa foi aplicada por conveniência a organizações que apresentaram como característica em comum, áreas ou atividades intensas em conhecimento. Ou seja, nestas organizações, o conhecimento desempenha um papel central na criação, uso, desenvolvimento, gerenciamento ou comercialização dos produtos ou serviços. Em meio a este cenário, a pesquisa foi aplicada às empresas prestadoras de serviços, indústrias e algumas instituições de ensino da Serra Gaúcha, no Rio Grande do Sul.

O Rio Grande do Sul apresenta o quinto maior PIB do Brasil, com a produção de R\$331,1 bilhões em bens e serviços, correspondendo a quase $7 \%$ da produção nacional (INSTITUTO BRASILEIRO DE GEOGRAFIA E ESTATÍSTICA - IBGE, 2013). As principais economias do estado se concentram na agricultura (soja, trigo, arroz e milho), na pecuária e na indústria (de couro e calçados, alimentícia, têxtil, madeireira, metalúrgica e química) (ATLAS SOCIOECONÔMICO, 2016).

A Região Metropolitana da Serra Gaúcha, criada pela Lei Complementar no 14.293, de 29 de agosto de 2013 (RIO GRANDE DO SUL, 2013), envolve é composta pelos municípios de Antônio Prado, Bento Gonçalves, Carlos Barbosa, Caxias do Sul, Farroupilha, Flores da Cunha, Garibaldi, Ipê, São Marcos, Nova Pádua, Monte Belo do Sul, Santa Teresa e Pinto Bandeira. A Serra Gaúcha que apresenta o terceiro maior PIB do estado, ficando atrás apenas da região Metropolitana de Porto Alegre e do Vale dos Sinos (IBGE, 2010). Entretanto, nos dados divulgados pela Federação das Indústrias do Rio Grande do Sul (FIERGS, 2014), em relação à empregabilidade da indústria, a cidade de Caxias do Sul é a cidade com maior número de trabalhadores (81 mil trabalhadores), correspondendo a $11,2 \%$ do total do estado.

Considerando os dados acima citados, a pesquisa ambientou-se em empresas do Rio Grande do Sul, mais especificamente da Serra Gaúcha, sendo que a coleta de dados foi realizada por meio da aplicação de questionários estruturados (MALHOTRA; BIRKS; WILLS, 2012) que foram aplicados através do autopreenchimento (eletrônico e impresso). Na elaboração do instrumento de coleta de dados, para a operacionalização da escala, foi definida a utilização de uma escala do tipo Likert de sete pontos, com possibilidade de respostas com extremos variando de " 1 . Discordo Totalmente" a "7. Concordo Totalmente" (BEARDEN; NETEMEYER; HAWS, 2011; NETEMEYER; BEARDEN; SHARMA, 2003). O 
link da pesquisa (questionário) foi disponibilizado por e-mail, e respondido através do método de auto-preenchimento no google docs ${ }^{\circ}$ (FOWLER Jr., 2009; FINK, 2013; MALHOTRA; BIRKS; WILLS, 2012). Para algumas empresas foram realizados contatos através de ligações telefônicas para o envio do e-mail e link da pesquisa (MALHOTRA; BIRKS; WILLS, 2012).

As escalas utilizadas foram operacionalizadas a partir de estudos anteriores, conforme mostra a Figura 1. A escala de criação do conhecimento de Chou e Wang (2003) foi citada por Choo e Alvarenga Neto (2010) em um estudo que abordou a expansão do conceito ba e as diferentes aplicações e desenvolvimento do assunto, num período considerado entre 1991 a 2009. Optou-se por esta escala quantitativa para mensurar a criação do conhecimento por existir relação com os objetivos deste trabalho. Com relação à escala de satisfação com o ambiente construído, foi utilizada uma escala de avaliação pós-ocupação do ambiente; esta base de dados utilizada pelo Centro de Ambiente Construído (Center for the Built Environment - CBE) da Universidade da Califórnia (Berkeley) (KIM et al., 2013).

Uma vez elaborado e estruturado o questionário de pesquisa, foi procedida à validação de conteúdo, também conhecida como validade de face (KINNEAR; TAYLOR, 1996), pela qual o instrumento de coleta de dados foi submetido a dois experts da área. A coleta de dados foi realizada no mês de novembro de 2014 a março de 2015 (FOWLER Jr., 2009; FINK, 2013). Foram aplicados 127 questionários, tabulados os dados e realizadas as verificações de consistência. Foi utilizado o software $P A S W \circledR$ Statistics 20.0. Deste total, não houve falta de consistência (missing values) e não houve observações atípicas (outliers). Um questionário (28) foi eliminado por apresentar valor $>3,0$ para o $Z$ Score (HAIR Jr. et al., 2010).

Após as verificações dos dados e das correções das inconsistências, a análise de dados foi realizada. Com o exame do conjunto de relações das variáveis interdependentes que integram a satisfação com o ambiente construído e a validação das variáveis dependentes que compõem a criação de conhecimento, as análises foram feitas através do exame do conjunto de relações da variável dependente. Foi realizado também a medida de adequação da amosta de Kaiser-Meyer-Olkin (KMO) e a confiabilidade das escalas a partir do Alfa de Cronbach (MALHOTRA; BIRKS; WILLS, 2012; JOHNSON; WICKERN, 2007; HAIR Jr. et al., 2010).

Além disso, a regressão linear foi utilizada para identificar quanto a satisfação com o ambiente construído explicou ou causou impacto sobre a criação do conhecimento. Esta técnica estatística analisa as relações associativas entre uma variável métrica dependente e a variável independente (AFIFI; MAY; CLARK, 2012; MALHOTRA; BIRKS; WILLS, 2012). A regressão linear foi elaborada com base nas médias do fator independente em relação à média da variável dependente. A seleção do método de análise descriminante passo-a-passo (stepwise discriminant analysis) consistiu em identificar os subconjuntos ou categorias, de modo 
a adicionar ou remover as variáveis em cada etapa (MALHOTRA; BIRKS; WILLS, 2012).

\section{Análise dos resultados}

A pesquisa foi aplicada às organizações, em ambientes de escritórios, que apresentaram como característica em comum, áreas intensas em conhecimento. Ou seja, nestas organizações, o conhecimento desempenha um papel central na criação, uso, desenvolvimento, gerenciamento ou comercialização dos produtos ou serviços. Em meio a este cenário, são apresentados os cargos desempenhados por cada respondente de acordo com a Tabela 1.

Tabela 1 - Cargo dos respondentes

\begin{tabular}{lcc}
\hline Cargo dos Respondentes & $\begin{array}{c}\text { Número de } \\
\text { Respondentes }\end{array}$ & $\%$ \\
\hline \hline administrador & 1 & $0,79 \%$ \\
\hline agente de pós venda & 1 & $0,79 \%$ \\
\hline analista ti & 4 & $3,15 \%$ \\
\hline analista de projeto & 1 & $0,79 \%$ \\
\hline analista de marketing & 9 & $7,09 \%$ \\
\hline analista de vendas & 2 & $1,57 \%$ \\
\hline analista de crédito & 2 & $1,57 \%$ \\
\hline analista de engenharia & 2 & $1,57 \%$ \\
\hline analista de inteligência de vendas & 2 & $1,57 \%$ \\
\hline analista de engenharia de produto & 1 & $0,79 \%$ \\
\hline analista de negócios & 2 & $1,57 \%$ \\
\hline analista de treinamento & 1 & $0,79 \%$ \\
\hline arquiteto & 22 & $17,32 \%$ \\
\hline assistente administrativo & 1 & $0,79 \%$ \\
\hline auxiliar administrativo & 3 & $2,36 \%$ \\
\hline auxiliar de vendas & 1 & $0,79 \%$ \\
\hline consultor de vendas & 6 & $4,72 \%$ \\
\hline consultor de atendimento & 1 & $0,79 \%$ \\
\hline contador & 1 & $0,79 \%$ \\
\hline contas a pagar e a receber & 1 & $0,79 \%$ \\
\hline coordenador & 2 & $1,57 \%$ \\
\hline coordenador de pedagógica & 1 & $0,79 \%$ \\
\hline coordenador de curso de graduação & 1 & $0,79 \%$ \\
\hline coordenador de curso de pós-graduação & 1 & $0,79 \%$ \\
\hline coordenação da produção & 2 & $1,57 \%$ \\
\hline corretor de seguros & 1 & $0,79 \%$ \\
\hline customizador de produto pleno & 1 & $0,79 \%$ \\
\hline desenvolvedor & 2 & $1,57 \%$ \\
\hline designer de produtos & 1 & $0,79 \%$ \\
\hline designer gráfico & 4 & $3,15 \%$ \\
\hline desenvolvedor de programas & 1 & $0,79 \%$ \\
\hline diretor comercial & 2 & $1,57 \%$ \\
\hline diretor de criação & 1 & $0,79 \%$ \\
\hline diretor empresarial & 1 & $0,79 \%$ \\
\hline
\end{tabular}


Influência do ambiente físico de trabalho na criação do conhecimento nas organizações
Ana Paula Graciola; Suélen Bebber; Fernanda Pauletto D'Arrigo; Ana Cristina Fachinelli; Gabriel Sperandio Milan;Deonir de Toni

\begin{tabular}{lll}
\hline diretor de operações & 1 & $0,79 \%$ \\
\hline engenheiro & 3 & $2,36 \%$ \\
\hline engenheiro civil & 1 & $0,79 \%$ \\
\hline engenheiro de produção & 1 & $0,79 \%$ \\
\hline engenheiro mecânico & 1 & $0,79 \%$ \\
\hline gerente de contas & 1 & $0,79 \%$ \\
\hline gerente comercial & 1 & $0,79 \%$ \\
\hline gerente industrial & 2 & $1,57 \%$ \\
\hline gerente marketing & 1 & $0,79 \%$ \\
\hline gerente projetista & 1 & $0,79 \%$ \\
\hline gerente & 2 & $1,57 \%$ \\
\hline gerente jurídico e de governança & 1 & $0,79 \%$ \\
\hline gestão de produtos & 1 & $0,79 \%$ \\
\hline mídia e relações públicas & 1 & $0,79 \%$ \\
\hline projetista & 1 & $0,79 \%$ \\
\hline programador & 2 & $1,57 \%$ \\
\hline pcp & 1 & $0,79 \%$ \\
\hline professor universitário & 1 & $0,79 \%$ \\
\hline relações humanas & 1 & $0,79 \%$ \\
\hline secretária executivo & 2 & $1,57 \%$ \\
\hline supervisor (contas pessoa jurídica) & 1 & $0,79 \%$ \\
\hline suporte & 1 & $0,79 \%$ \\
\hline telemarketing & 1 & $0,79 \%$ \\
\hline tesoureira & 1 & $0,79 \%$ \\
\hline missing value & 12 & $9,45 \%$ \\
\hline \hline Total & $\mathbf{1 2 7}$ & $\mathbf{1 0 0 , 0 0 \%}$ \\
\hline
\end{tabular}

Fonte: Dados da pesquisa.

Os dados foram coletados em organizações localizadas no estado do Rio Grande do Sul. Os respondentes possuem como característica em comum o fato de trabalharem em áreas intensas em conhecimento, em que o conhecimento desempenha papel principal no desenvolvimento, gerenciamento e comercialização de produtos e serviços. No total 127 respondentes participaram da pesquisa, conforme demonstra a Tabela 2.

\section{Tabela 2 - Perfil dos respondentes}

\begin{tabular}{l|c|c}
\hline \multicolumn{1}{c|}{ Características Demográficas } & Casos $(\mathbf{n}=\mathbf{1 2 7})$ & Porcentagem (\%) \\
\hline Sexo & 61 & 48.4 \\
\hline Masculino & 64 & 50.8 \\
\hline Feminino & 1 & 0.8 \\
\hline Missing & 28 & 22.2 \\
\hline Idade & 55 & 43.7 \\
\hline $18-25$ & 22 & 17.5 \\
\hline $26-35$ & 11 & 8.7 \\
\hline $36-45$ & 9 & 7.1 \\
\hline $46-55$ & 1 & 0.8 \\
\hline $56-65$ & \multicolumn{2}{|l}{} \\
\hline Missing & 2 & 1.6 \\
\hline Grau de Escolaridade & 22 & 17.5 \\
\hline Ensino médio completo & 46 & 36.5 \\
\hline Ensino superior incompleto & \multicolumn{3}{|l}{} \\
\hline Ensino superior completo &
\end{tabular}


Influência do ambiente físico de trabalho na criação do conhecimento nas organizações
Ana Paula Graciola; Suélen Bebber; Fernanda Pauletto D'Arrigo; Ana Cristina Fachinelli; Gabriel Sperandio Milan;Deonir de Toni

\begin{tabular}{l|c|c}
\hline Pós-graduação Incompleto & 18 & 14.3 \\
\hline Pós-graduação Completo & 29 & 2.3 \\
\hline Mestrado & 7 & 5.6 \\
\hline Doutorado & 2 & 1.6 \\
\hline Experiência de trabalho na empresa (anos): & \multicolumn{2}{|c}{} \\
\hline menos de 1 ano & \multicolumn{2}{|c}{} \\
\hline entre 1 e 2 anos & 18 & 14,3 \\
\hline entre 2 e 3 anos & 27 & 21,4 \\
\hline entre 3 e 4 anos & 12 & 9,5 \\
\hline entre 4 e 5 anos & 11 & 8,7 \\
\hline entre 5 e 10 anos & 31 & 24,6 \\
\hline entre 10 e 15 anos & 10 & 7,9 \\
\hline mais que 15 anos & 9 & 7,1 \\
\hline Tempo Permanência sentado sem levantar da cadeira & 3 & 2.4 \\
\hline 15 minutos & 5 & 12.7 \\
\hline 45 minutos & 16 & 7.1 \\
\hline Uma hora & 9 & 1.6 \\
\hline Uma hora e meia & 2 & 16.7 \\
\hline Mais de uma hora e meia & 21 & 18.3 \\
\hline Duas horas & 23 & 35.7 \\
\hline Três horas & 25 & 1.6 \\
\hline Quatro horas & 25 \\
\hline Missings & & \\
\hline
\end{tabular}

Fonte: Dados da pesquisa.

Com base nas variáveis analisadas, os atributos validados foram classificados em cinco dimensões ou fatores. Dentre estas cinco dimensões, a satisfação com o ambiente construído (SAC) aparece como a dimensão mais importante; na sequência a criação de conhecimento (tecnologia) (CCA); a criação de conhecimento (tácito) (CCB); a criação de conhecimento (aprendizagem) (CCC) e a criação de conhecimento (interação) (CCD), fatores (dimensões) que foram validados (vide Tabela 3). A depuração das escalas foi realizada com a retirada de atributos com cargas fatoriais inferiores a 0,50 (HAIR Jr. et al., 2010; JOHNSON; WICKERN, 2007), resultando em cinco dimensões (fatores) com uma variância total explicada de 70,093\%.

Tabela 3 - Resultado para extração dos fatores (ou dimensões)

\begin{tabular}{c|c|c|c|c|c|c}
\hline \multirow{2}{*}{ Atributos } & \multicolumn{5}{|c|}{ Fatores ou Dimensões } & \multirow{2}{*}{ Comunalidades } \\
\cline { 2 - 5 } & $\mathbf{1}$ & $\mathbf{2}$ & $\mathbf{3}$ & $\mathbf{4}$ & $\mathbf{5}$ & 0,780 \\
\hline SAC11 & 0,825 & & & & & 0,752 \\
\hline SAC5 & 0,801 & & & & & 0,710 \\
\hline SAC7 & 0,767 & & & & & 0,671 \\
\hline SAC10 & 0,754 & & & & & 0,613 \\
\hline SAC9 & 0,711 & & & & & 0,600 \\
\hline SAC8 & 0,693 & & & & & 0,574 \\
\hline SAC6 & 0,638 & & & & & 0,687 \\
\hline CC2A & & 0,792 & & & & 0,742 \\
\hline CC4A & & 0,783 & & & & 0,651 \\
\hline CC3A & & 0,746 & & & & 0,690 \\
\hline CC1A & & 0,744 & & & & 0,582 \\
\hline CC5A & & 0,622 & & & & 0,862 \\
\hline CC8B & & & 0,902 & & & \\
\hline
\end{tabular}


Influência do ambiente físico de trabalho na criação do conhecimento nas organizações
Ana Paula Graciola; Suélen Bebber; Fernanda Pauletto D'Arrigo; Ana Cristina Fachinelli; Gabriel Sperandio Milan;Deonir de Toni

\begin{tabular}{c|c|c|c|c|c|c}
\cline { 4 - 6 } CC7B & & & 0,857 & & & 0,775 \\
\hline CC9B & & & 0,760 & & & 0,697 \\
\hline CC12C & & & & 0,858 & & 0,821 \\
\hline CC13C & & & & 0,614 & & 0,668 \\
\hline CC14D & & & & & 0,855 & 0,763 \\
\hline CC15D & & & & & 0,568 & 0,680 \\
\hline Autovalor & $\mathbf{6 , 9 4 5}$ & $\mathbf{2 , 4 2 6}$ & $\mathbf{1 , 7 7 8}$ & $\mathbf{1 , 1 6 1}$ & $\mathbf{1 , 0 0 8}$ & \% de Variância Acumulada \\
\hline $\begin{array}{c}\text { \% de Variância } \\
\text { Explicada }\end{array}$ & $\mathbf{3 6 , 5 5 3}$ & $\mathbf{1 2 , 7 7}$ & $\mathbf{9 , 3 5 5}$ & $\mathbf{6 , 1 0 9}$ & $\mathbf{5 , 3 0 6}$ & $\mathbf{7 0 , 0 9 3}$ \\
\hline
\end{tabular}

Fonte: Dados da pesquisa.

Nota: Teste de Esfericidade de Bartlett: Qui-quadrado: 1274,380; KMO: 0,835; GL: 171,000; sig.: 0,000.

Dos 27 atributos inicialmente avaliados, 19 deles ficaram retidos. Com o intuito de facilitar o entendimento sobre estes achados da pesquisa, a Quadro 1 relaciona tanto as dimensões responsáveis pela satisfação com o ambiente construído e a criação do conhecimento, quanto os seus respectivos atributos. Porém, foi contatado que o fator criação de conhecimento (interação) apresentou valores de Alfa de Cronbach baixo e, portanto, será eliminado, por indicar baixa correlação entre os itens desse fator e também baixa confiabilidade (HAIR Jr. et al., 2010).

Quadro 1 - Dimensões da satisfação com o ambiente construído e a criação do conhecimento

\begin{tabular}{|c|c|c|c|}
\hline Dimensões & $\begin{array}{l}\text { Alfa de } \\
\text { Cronbach }\end{array}$ & & Atributos Relacionados às Dimensões \\
\hline \multirow{7}{*}{$\begin{array}{l}\text { Satisfação com o } \\
\text { Ambiente Construído }\end{array}$} & \multirow{7}{*}{0,893} & SAC11 & $\begin{array}{l}\text { Considerados todos os fatores, estou satisfeito com meu } \\
\text { espaço de trabalho pessoal. }\end{array}$ \\
\hline & & SAC5 & $\begin{array}{l}\text { Estou satisfeito com a quantidade de espaço físico } \\
\text { disponível para cada indivíduo. }\end{array}$ \\
\hline & & SAC7 & $\begin{array}{l}\text { Estou satisfeito com o conforto do mobiliário onde } \\
\text { trabalho. }\end{array}$ \\
\hline & & SAC10 & $\begin{array}{l}\text { Estou satisfeito com a limpeza em geral de toda a } \\
\text { edificação. }\end{array}$ \\
\hline & & SAC9 & $\begin{array}{l}\text { Estou satisfeito com as cores e texturas nos pisos, } \\
\text { mobiliários, paredes e tetos. }\end{array}$ \\
\hline & & SAC8 & $\begin{array}{l}\text { Estou satisfeito com a possibilidade de ajustar (mover) os } \\
\text { mobiliários/equipamentos para atender minhas } \\
\text { necessidades. }\end{array}$ \\
\hline & & SAC6 & $\begin{array}{l}\text { Estou satisfeito com a facilidade de interação com os } \\
\text { colegas de trabalho. }\end{array}$ \\
\hline \multirow{2}{*}{$\begin{array}{l}\text { Criação do } \\
\text { Conhecimento } \\
\text { (Tecnologia) }\end{array}$} & \multirow[t]{2}{*}{0,854} & $\mathrm{CC} 2 \mathrm{~A}$ & $\begin{array}{l}\text { Os sistemas de informação apresentam programas de } \\
\text { simulação. }\end{array}$ \\
\hline & & $\mathrm{CC} 4 \mathrm{~A}$ & $\begin{array}{l}\text { Os técnicos que devenvolvem a tecnologia de informação } \\
\text { trocam ideias com quem utiliza o sistema para melhorar a } \\
\text { tecnologia da informação. }\end{array}$ \\
\hline
\end{tabular}


Influência do ambiente físico de trabalho na criação do conhecimento nas organizações
Ana Paula Graciola; Suélen Bebber; Fernanda Pauletto D'Arrigo; Ana Cristina Fachinelli; Gabriel Sperandio Milan;Deonir de Toni

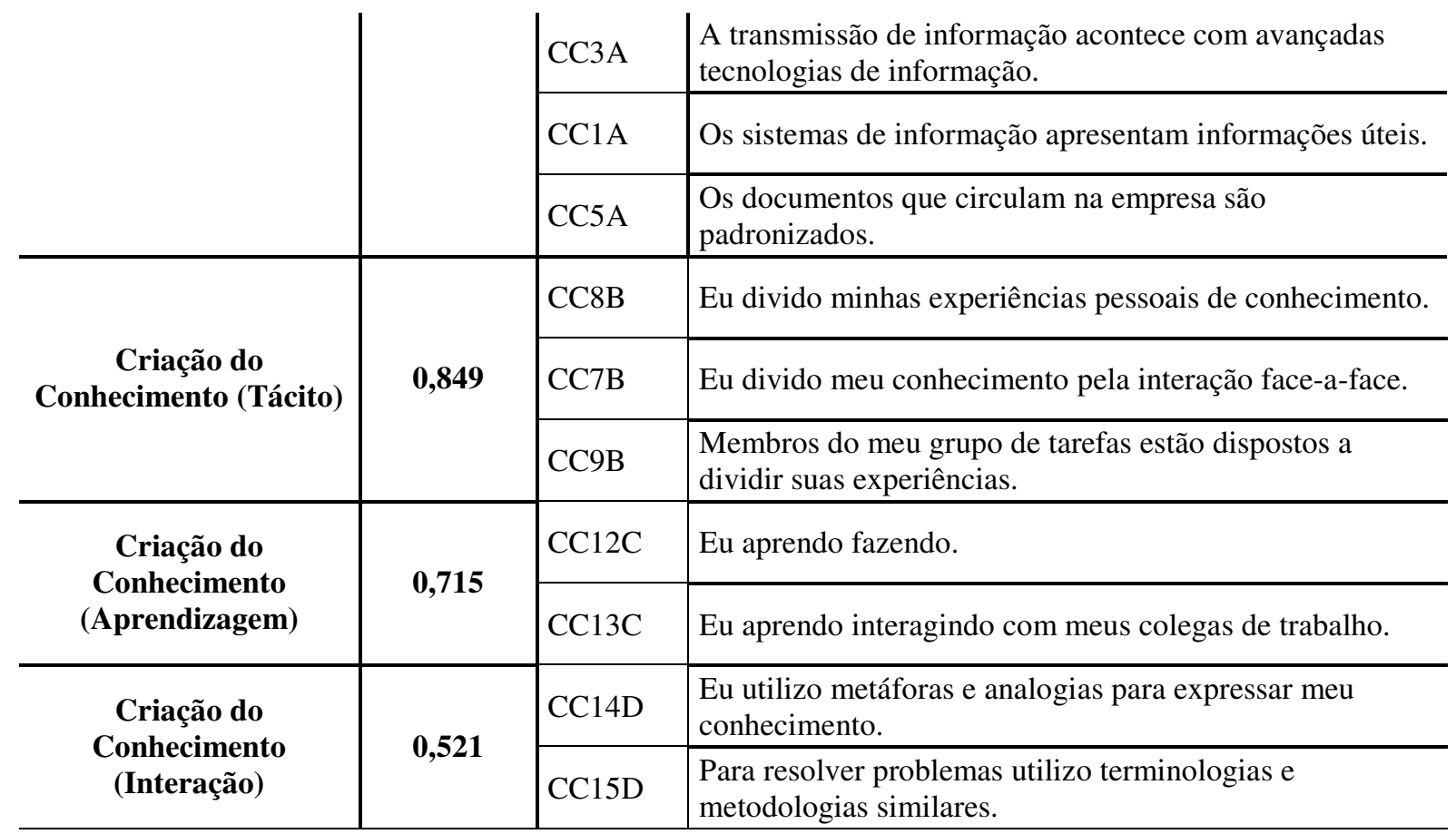

Fonte: Dados da pesquisa.

A regressão linear verificou o quanto a dimensão da satisfação com o ambiente construído impacta sobre as quatro dimensões da criação do conhecimento. Foi encontrado que a satisfação com o ambiente construído apresenta maior impacto sobre a criação de conhecimento, nas áreas da tecnologia e aprendizagem. Além do mais, a satisfação com o ambiente construído também apresenta significância para a criação do conhecimento tácito e a interação, no entanto, com menor influência em decorrência dos valores menores encontrados no $\mathrm{R}^{2}$ e Beta $(\beta)$, conforme Tabela 4, que é elucidativa.

Tabela 4 - Dimensões da satisfação com o ambiente construído e a criação do conhecimento

\begin{tabular}{c|c|c|c|c}
\hline Indicadores & $\begin{array}{c}\text { Criação do } \\
\text { Conhecimento } \\
\text { (Tecnologia) }\end{array}$ & $\begin{array}{c}\text { Criação do } \\
\text { Conhecimento } \\
\text { (Tácito) }\end{array}$ & $\begin{array}{c}\text { Criação do } \\
\text { Conhecimento } \\
\text { (Aprendizagem) }\end{array}$ & $\begin{array}{c}\text { Criação do } \\
\text { Conhecimento } \\
\text { (Interação) }\end{array}$ \\
\hline $\mathrm{R}^{2}$ & 0,333 & 0,091 & 0,106 & 0,041 \\
\hline $\begin{array}{c}\text { Satisfação com o Ambiente } \\
\text { Construído }\end{array}$ & $\beta=0,577$ & $\beta=0,301$ & $\beta=0,326$ & $\beta=0,202$ \\
\hline Significância & 0,000 & 0,001 & 0,000 & 0,023 \\
\hline
\end{tabular}

Fonte: Dados da pesquisa.

Através do teste $t^{1}$ da ANOVA, de acordo com a Tabela 5, foi possível comparar 0 quanto as médias das dimensões em análise apresentam significância com relação ao perfil dos respondentes. Portanto, foi constatado que o tempo de permanência sentado, sem

\footnotetext{
${ }^{1}$ Teste $t$ - Teste para avaliar a significância estatística da diferença entre duas médias amostrais para uma única variável dependente. O teste $t$ é um caso especial da ANOVA para dois grupos ou níveis de uma variável de tratamento (HAIR Jr. et al., 2010).
} 
levantar da cadeira, influência na satisfação com o ambiente construído. Outro ponto observado foi a influência da faixa etária na criação do conhecimento através do uso da tecnologia. E por fim, a experiência de trabalho interfere na criação do conhecimento através da interação, ou seja, quanto maior a experiência de trabalho melhor as interações para a criação do conhecimento.

Tabela 5 - Comparação entre as médias das dimensões em análise e o perfil dos respondentes

\begin{tabular}{|c|c|c|c|c|}
\hline Médias & Faixa etária & Nível Educacional & $\begin{array}{c}\text { Experiência de } \\
\text { trabalho }\end{array}$ & $\begin{array}{c}\text { Tempo } \\
\text { permanência } \\
\text { sentado (sem } \\
\text { levantar) } \\
\end{array}$ \\
\hline \multirow{2}{*}{$\begin{array}{c}\text { Satisfação com o } \\
\text { ambiente } \\
\text { construído }\end{array}$} & $\mathrm{F}=0,895$ & $\mathrm{~F}=1,639$ & $\mathrm{~F}=1,808$ & $\mathrm{~F}=\mathbf{2 , 1 3 5}$ \\
\hline & Sig. $=0,469$ & Sig. $=0,142$ & Sig. $=0,092$ & Sig. $=0,045$ \\
\hline \multirow{2}{*}{$\begin{array}{c}\text { Criação do } \\
\text { conhecimento } \\
\text { (tecnologia) }\end{array}$} & $F=2,620$ & $\mathrm{~F}=1,647$ & $\mathrm{~F}=1,459$ & $\mathrm{~F}=1,673$ \\
\hline & Sig. $=0,038$ & Sig. $=0,140$ & Sig. $=0,189$ & Sig. $=0,122$ \\
\hline \multirow{2}{*}{$\begin{array}{c}\text { Criação do } \\
\text { Conhecimento } \\
\text { (tácito) }\end{array}$} & $\mathrm{F}=2,161$ & $\mathrm{~F}=0,290$ & $\mathrm{~F}=1,076$ & $\mathrm{~F}=0,977$ \\
\hline & Sig. $=0,078$ & Sig. $=0,941$ & Sig. $=0,383$ & Sig. $=0,452$ \\
\hline \multirow{2}{*}{$\begin{array}{c}\text { Criação do } \\
\text { conhecimento } \\
\text { (aprendizagem) }\end{array}$} & $\mathrm{F}=0,567$ & $\mathrm{~F}=1,297$ & $\mathrm{~F}=0,389$ & $\mathrm{~F}=1,681$ \\
\hline & Sig. $=0,687$ & Sig. $=0,264$ & Sig. $=0,907$ & Sig. $=0,120$ \\
\hline \multirow{2}{*}{$\begin{array}{c}\text { Criação do } \\
\text { conhecimento } \\
\text { (interação) }\end{array}$} & $\mathrm{F}=0,804$ & $\mathrm{~F}=1,880$ & $F=3,209$ & $\mathrm{~F}=1,248$ \\
\hline & Sig. $=0,525$ & Sig. $=0,090$ & Sig. $=0,004$ & Sig. $=0,282$ \\
\hline
\end{tabular}

Fonte: Dados da pesquisa.

\section{Considerações finais}

O presente estudo teve como objetivo identificar a influência da satisfação com o ambiente construído no processo de criação do conhecimento. O estudo foi ambientado em empresas intensivas em conhecimento do Rio Grande do Sul. Ao tratar o campo da criação do conhecimento, o estudo adotou os conceitos de Chou e Wang (2003) ao contemplar quatro áreas para a criação do conhecimento, sendo elas: a criação de (i) conhecimento tácito, (ii) explícito relacionado a aprendizagem, (iii) a interação e (iv) a participação da tecnologia na criação de conhecimento. Para mensurar a influência do ambiente construído, levou-se em consideração o estudo de Kim et al. (2013), o qual aborda a satisfação com o espaço físico de trabalho, estética, conforto, limpeza, organização e a interação entre os colegas de trabalho.

Os resultados apontaram que das quatro áreas que representam a criação do conhecimento, a satisfação com o ambiente construído apresentou maior influencia no uso da tecnologia para a criação do conhecimento. Consoante isso, melhorar a satisfação com o ambiente construído propicia os encontros entre as pessoas, facilita 0 compartilhamento das informações e contribui para a criação do conhecimento nas três áreas abordadas neste estudo (tecnológica, tácita, 
aprendizagem). Outro achado da pesquisa está na relação positiva entre a criação de conhecimento tácito e a satisfação com o ambiente construído, assim a criação do conhecimento tácito é influenciada pelo ambiente construído.

Em paralelo aos objetivos propostos, foi possível constatar a existência de valor para o trabalho agregado, que se encontra justamente nesta comparação entre o ambito econômico (tangível) de melhoramentos do espaço construído nos escritórios, o custo do ambiente construído. Em contrapartida, quanto melhor for o espaço construído, maior será o valor de trabalho para a criação de novos conhecimentos nas organizações.

\section{Referências}

AFIFI, A.; MAY, S.; CLARK, V. A. Practical multivariate analysis. $5^{\text {th }}$ edition. Boca Raton: Taylor \& Francis Group, 2012.

ALAVI, M.; LEINER, D. E. Knowledge management and knowledge management systems: conceptual foundations and research issues, MIS Quartely, v. 25, n. 1, p. 107-136, 2001.

ALLEN, T. J. Managing the Flow of Technology: technology transfer and the dissemination of technological information within the $R \& D$ organization. Cambridge, Massachusetts; London, England: The MIT Press, 1977.

ALLEN, T. J.; HENN, G. W. The organization and architecture of innovation: managing of Flow of technology. Oxford, UK: Elsevier Inc., 2007.

CHOO, C. W.; ALVARENGA NETO, R. C. D. Beyond the ba: managing enabling contexts in knowledge organizations. Journal of Knowlegde Management, v. 14, n. 4, p. 592-610, 2010.

ATLAS SOCIOECONÔMICO. Participação do PIB Estadual. Disponível em: <http://www.atlassocioeconomico.rs.gov.br/conteudo.asp?cod_menu_filh $\mathrm{o}=818 \&$ cod_menu $=817 \&$ tipo_menu $=$ ECONOMIA\&cod_conteudo $=1468>$.

Acesso em: 16 fev. 2016.

BEARDEN, W. O.; NETEMEYER, R. G.; HAWS, K. L. Handbook of marketing scales: multi-item measures for marketing and consumer behavior research. $3^{\text {rd }}$ edition. Thousand Oaks: Sage Publications, 2011.

BURGE, P. S. et al. Sick building syndrome: a study of 4373 office workers. Annals Occupational Hygiene, v. 31, p. 493-504, 1987.

CARLOPIO, J. R. Construct validity of a physical work environment satisfaction questionnaire. Journal of Occupational Health Psychology, v. 1, n. 3, p. 330-344, 1996.

CHOU, S. W; WANG, S. J. Quantifying 'ba': an investigation of the variables that are pertinent to knowledge creation. Journal of Information Science, v. 29, n. 3, p. 167-180, 2003. 
CONGDON, C.; FLYNN, D.; REDMAN, M. Balancing "We" and "Me": the best collaborative spaces also support solitude. Harvard Business Review, v. 92, n. 10, p. 37-43, 2014.

DOLE, C.; SCHUROEDER, R. G. The impact of various factors on the personality, job satisfaction with the office environment. In: WINEMAN, J. D. (Ed.). Behavioral issues in office design. New York: Van Nostrand Reinhold, 1986. p. 85-108.

DRUCKER, P. Knowledge-worker productivity: the biggest challenge. California Management Review, v. 41, p. 79-94, 1999.

EDVINSSON, L.; MALONE, M. S. Capital intelectual: descobrindo o valor real de sua empresa pela identificação de seus valores internos. São Paulo: Makron Books, 1998.

FAYARD, P. O inovador modelo japonês de gestão do conhecimento. Porto Alegre: Bookman, 2010.

FEDERAÇÃO DAS INDÚSTRIAS DO RIO GRANDE DO SUL - FIERGS. Estudos Econômicos do RS - 2014. Disponível em: <http://www.fiergs.org.br/pt-br/economia/estudos-econ\%C3\%B4micosdo-rs>. Acesso em: 16 fev. 2016.

FINK, A. How to conduct surveys: a step-by-step guide. 5th edition. Thousand Oaks: Sage Publications, 2013.

FISK, W. Health and productivity gains from better indoor environment and their relationship with building energy efficiency. Annu Rev Energy Environment, v. 25, p. 537, 566, 2000.

FOWLER Jr., F. J. Survey research methods. $4^{\text {th }}$ edition. Thousand Oaks: Sage Publications, 2009.

GUPTA, J. ND; SHARMA, S. K. (Ed.). Creating knowledge based organizations. New York: Igi Global, 2004.

GUPTA, S.; KHARE, M.; GOYAL, R. Sick building syndrome: a case study in a multistory centrally air-conditioned building in the Delhi City. Building and Environment, v. 42, n. 8, p. 2797-2809, 2007.

HAIR Jr., J. F. et al. Multivariate data analysis. $7^{\text {th }}$ edition. New Jersey: Pearson, 2010.

HEDGE, A.; STERLING, E. M.; STERLING, T. D. Building illness indices based on questionnaire responses. Proceeding IAQ'86-Managing Indoor Air for Health and Energy conservation. Atlanta, GA, 1986. p. 31-43.

HESKETT, J. L. et al. Putting the service-profit chain to work. Harvard Business Review - Business Classics: Fifteen Key Concepts for Managerial Success, Boston, Harvard Business School Publishing, p. 110-120, 1994.

HUBER, G. P. Organizational learning: the contributing process and the literatures. Organization Science, v. 2, n. 1, p. 88-115, 1991. 
HUMPHREYS, M. A.; NICOL, J. F. Self-assessed productivity and the office environment: monthly surveys in five European countries. ASHRAE Transactions, v. 113, n. 1, p. 606-616, 2007.

INSTITUTO BRASILEIRO DE GEOGRAFIA E ESTATÍSTICA - IBGE. Estados@. Disponível em: <http://www.ibge.gov.br/estadosat/>. Acesso em: 15 fev. 2016.

JOHNSON, R.A.; WICKERN, D.W. Applied multivariate statistical analysis. $6^{\text {th }}$ edition. Upper Saddle River: Pearson / Prentice Hall, 2007.

JONES, A. P. Indoor air quality and hearth. Atmosphere Environment, v. 33, n. 28 , p. $4.535-4.564,1999$.

$\mathrm{KIM}$, J. et al. Gender differences in office occupant perception of indoor environmental quality (IEQ). Building and Environment, v. 70, p. 245-256, 2013.

KINNEAR, T. C.; TAYLOR, J. R. Marketing research: an applied approach. $5^{\text {th }}$ edition. New York: McGraw-Hill, 1996.

KOGUT, B.; ZANDER, U. Knowledge of the firm, combinative capabilities, and the replication of technology. Organization science, v. 3, n. 3, p. 383397, 1992.

LEAMAN, A.; BORDASS, B. Are users more tolerant of 'green' buildings? Building Research \& Information, v. 35, n. 6, p. 662-673, 2007.

LEE, Y. S. Expectations of employees toward the workplace and environmental satisfaction. Facilities, v. 24, n. 9/10, p. 343-353, 2006.

RIO GRANDE DO SUL. Lei Complementar no 14.293, de 29 de agosto de 2013. Diário Oficial da Assembléia Legislativa, Porto Alegre, RS, 12 set. 2013.

em:

<http://proweb.procergs.com.br/Diario/DA20130912-01-

100000/EX20130912-01-100000-PLC-231-2013.pdf>. Acesso em: 3 mar. 2016.

LEVITT, B.; MARCH, J. G. Organizational learning. Annual review of sociology, v. 14, p. 319-340, 1988.

MALHOTRA, N. K.; BIRKS, D.; WILLS, P. Marketing research: applied approach. $4^{\text {th }}$ edition. New York: Pearson, 2012.

MOLHAVE, L. The sick buildings. Proceedings of the $4^{\text {th }}$ International Conference on Indoor Air Quality and Climate, Berlin, n. 2, p. 469-473, 1987.

NETEMEYER, R. G.; BEARDEN, W. O.; SHARMA, S. Scaling procedures: issues and applications. Thousand Oaks: Sage Publications, 2003.

NONAKA, I.; KONNO, N. The concept of "ba": building foundation for knowledge creation, California Management Review, v. 40, n. 3, p. 40-54, 1998. 
NONAKA, I.; TAKEUCHI, H. The Knowledge-creating company: how japanese companies create the dynamics of innovation. New York: Oxford University Press, 1995.

NONAKA, I.; TOYAMA, R.; HIRATA, T. Teoria e casos de empresas baseadas no conhecimento: managing flow. Porto Alegre: Bookman, 2011.

NORBACK, D.; MICHEL, I.; WIDSTROM, U. Indoor air quality and personal factors related to the sick building syndrome. Scandinavian Journal of Work, Environment and Health, v. 16, p. 121-128, 1990.

RYAN, C. M.; MORROW, L. A. Dysfunctional buildings or dysfunctional people. Journal of Clinical and Consulting Psychology, v. 60, p. 220-224, 1992.

REMLER, D. K.; VAN RYZIN, G. G. Research methods in practice: strategies for description and causation. Thousand Oaks: Sage Publications, 2011.

STEWART, T. A. Capital intelectual: a nova vantagem competitiva das empresas. Rio de Janeiro: Campus, 1998.

VALJBORN, O. Building sickness syndrome: a guide to approach a complaint building. Copenhagen: NIVA Course on Sick Building Syndrome, 1989.

VAN DER VOORDT, T. J. M. Productivity and employee satisfaction in flexible workplaces. Journal of Corporate Real Estate, v. 6, n. 2, p. 133$148,2004$.

VAN DER VOORDT, T.J.M.; VAN WEGEN, H.B.R. Arquitetura sob o olhar do usuário. São Paulo: Oficina de textos, 2013.

WABER, B.; MAGNOLFI, J.; LINDSAY, G. Workspaces that move people. Harvard Business Review, v. 92, n. 10, p. 54 - 63, 2014.

WARGOCKI, P. The effects of outdoor air supply rate in an office on perceived air quality, sick building syndrome (SBS) symptoms and productivity. Indoor Air, v. 10, n.. 4, p. 222-236, 2000.

ZAHRA, S. A.; GEORGE, G. Absorptive capacity: a review, reconceptualization, and extension. Academy of management review, $\mathrm{V}$. 27, n. 2, p. 185-203, 2002. 